\title{
Immune-based therapies in the management of multiple myeloma
}

\author{
Saurabh Zanwar $\mathbb{1}^{1,2}$, Bharat Nandakumar ${ }^{1}$ and Shaji Kumar $\mathbb{1}^{1,2}$
}

\begin{abstract}
Multiple myeloma (MM) is a clonal plasma cell malignancy affecting a predominantly elderly population. The continued development of newer therapies with novel mechanisms of action has reshaped the treatment paradigm of this disorder in the last two decades, leading to a significantly improved prognosis. This has in turn resulted in an increasing number of patients in need of therapy for relapsed/refractory disease. Immune-based therapies, including monoclonal antibodies, immune checkpoint inhibitors, and most promisingly, adoptive cellular therapies represent important therapeutic strategies in these patients due to their non-cross resistant mechanisms of actions with the usual frontline therapies comprising of immunomodulatory drugs (IMiDs) and proteasome inhibitors (PIs). The antiCD38 antibodies daratumumab and more recently isatuximab, with their excellent efficacy and safety profile along with its synergy in combination with IMiDs and PIs, are being increasingly incorporated in the frontline setting. Chimeric antigen receptor-T cell (CART) therapies and bi-specific T-cell engager (BiTE) represent exciting new options that have demonstrated efficacy in heavily pretreated and refractory MM. In this review, we discuss the rationale for use of immune-based therapies in MM and summarize the currently available literature for common antibodies and CAR-T therapies that are utilized in MM.
\end{abstract}

\section{Introduction}

Multiple myeloma (MM) is a disorder of clonal plasma cells with a median age at diagnosis of around 67 years and accounts for $\sim 10 \%$ of all newly diagnosed hematologic malignancies ${ }^{1}$. The incorporation of novel agents into upfront therapy and the introduction of maintenance approaches have led to a sustained improvement in the overall survival (OS) of patients with MM over the past two decades ${ }^{2}$. Unfortunately, MM remains incurable and relapse of the disease is common. The improvement in outcomes of patients with MM has also presented the challenge of treating an increasingly elderly population at relapse, making it important to have drugs with a better efficacy and safety profile to minimize toxicities ${ }^{3}$. A host of new agents have been approved for use in relapsed/ refractory MM (RRMM) and potentially the most revolutionary class of therapeutics that have been introduced

\footnotetext{
Correspondence: Shaji Kumar (Kumar.Shaji@mayo.edu)

${ }^{1}$ Division of Hematology, Mayo Clinic, Rochester, MN, USA

${ }^{2}$ Department of Internal Medicine, Mayo Clinic, Rochester, MN, USA
}

in the treatment paradigm of $\mathrm{MM}$ in the past few years include immune-based therapies targeting the malignant cell clone ${ }^{4,5}$. Immune-based therapy for treatment of MM is not a new concept. Allogenic stem cell transplant has been utilized as a treatment strategy in $M M$ and the presence of a graft versus myeloma effect points toward the efficacy of this approach ${ }^{6}$. The high rates of transplant-related mortality associated with allogenic transplant have made it a less preferred modality, but the potentially curative nature of this treatment makes it unique in the treatment landscape for $\mathrm{MM}^{7}$. Immune dysregulation is postulated to be centrally involved in the pathogenesis and disease progression in $\mathrm{MM}^{8}$. In this review, we discuss the rationale, targets, and evidence for immune-based therapies in treating MM.

\section{Immune dysregulation in MM}

\section{Role of immune checkpoints in pathogenesis of MM}

A normal cell-mediated immune response is driven by the interaction between antigen presenting cells (APC) 
and effector $\mathrm{T}$ cells, and is orchestrated by the dynamic balance between the activating and inhibitory signaling molecules and cytokines ${ }^{9}$. The B7-CD28 family of proteins, especially cytotoxic tumor lymphocyte antigen 4 (CTLA-4) and programmed cell death-1 (PD-1) are important co-inhibitory molecules that are expressed on $\mathrm{T}$-cells, B-cells, and natural killer (NK) cells ${ }^{10}$. These serve as important immune checkpoints and regulate the production of antigen-specific T-cells thus playing are an important role in maintaining immune tolerance ${ }^{11}$. Programmed cell death-ligand 1 (PD-L1), the ligand for PD-1 receptor, is expressed on APCs and its binding to PD-1 leads to suppression of $\mathrm{T}$-cell activation and immune response. Cytotoxic tumor lymphocyte antigen-4 competes with the co-stimulatory molecule CD28 in binding to CD80 and CD86 thereby limiting the production of antigen-specific T cells ${ }^{11}$. Similarly, the binding of PD-L1 to PD-1 leads to immune inhibition and escape of tumor cells from immune surveillance ${ }^{11}$. Both CTLA- 4 and PDL1 are shown to have increased expression in bone marrow milieu of patients with $\mathrm{MM}^{12,13}$.

Another important player involved in the immune regulation of $\mathrm{T}$ cells in $\mathrm{MM}$ is lymphocyte activation gene-3 (LAG3; CD223). The role of LAG3 as an immune checkpoint was identified in relation to its role in enhancing the function of regulatory $\mathrm{T}$ cells $\left(\mathrm{T}_{\text {regs }}\right)$ and inhibition of CD8 T-cells ${ }^{14}$. Like PD-1/PD-L1, LAG3 expression can prevent the development of autoimmunity but sustained LAG3 stimulation can be associated with Tcell exhaustion which can potentially contribute to immune escape ${ }^{10}$. Increased expression of LAG3 on $\mathrm{T}$ cells in the tumor milieu of MM has been noted in murine models and there is preclinical evidence of synergy between PD-1 and LAG3 inhibition, which could represent an important dual immune targeting strategy ${ }^{15}$. In a small study performed on 16 bone marrow specimens, patients with faster progression of smoldering MM had an expression of negative immune regulatory mediators including LAG3 expression on the T cells in the microenvironment and PD-L1 on the plasma cells ${ }^{16}$. A recent study also demonstrated that increased LAG3 transcript expression in T-cells postautologous transplant was associated with a worse outcome in patients with MM, suggesting potential role of targeting LAG3 in $\mathrm{MM}^{17}$. Other important immune checkpoints include Tcell immunoglobulin (TIM)-3 and T-cell immunoreceptor with Ig and ITIM (TIGIT) domains which are currently being studied as potential targets for therapy ${ }^{18}$.

\section{$T_{\text {reg-mediated immune suppression in } M M}$}

$\mathrm{T}_{\text {regs }}$ are involved in the maintenance of self-tolerance and prevention of autoimmunity by exerting an inhibitory effect on immune response through various mechanisms, including release of inhibitory interleukins (IL) like IL-10,
TGF- $\beta$, and IL-35 that leads to a state of T-cell anergy and immune paresis ${ }^{19,20}$. The bone marrow (BM) and peripheral blood in patients with $\mathrm{MM}$ and monoclonal gammopathy of undetermined significance show increased frequency of $\mathrm{T}_{\text {regs }}$ compared to that in patients of age-matched healthy controls ${ }^{21}$. Also, CTLA-4 is expressed on $\mathrm{T}_{\text {regs }}$ and plays a role in mediating the immune suppressive effects of $\mathrm{T}_{\text {regs }}{ }^{13}$. The $\mathrm{T}_{\text {regs }}$ are believed to play an important role in resistance to immune-mediated destruction in MM, making them potentially important drug targets ${ }^{22}$.

\section{BM microenvironment in immune dysfunction in MM}

The BM microenvironment is thought to play a central role in the development and progression of $\mathrm{MM}^{8}$. There is an extensive crosstalk between the BM stromal cells (BMSCs), BM endothelial cells and the MM cells which leads to the secretion of cytokines like hepatocyte growth factor (HGF), vascular endothelial growth factor, transforming growth factor- $\beta$, stromal cell-derived factor- $1 \alpha$ (SDF-1 $\alpha$ ) among others that promote myeloma cell sur$\mathrm{vival}^{23}$. Additionally, interleukin-6 is released from the interaction of myeloma cells with BMSCs and promotes survival of the malignant cells ${ }^{24}$. The change in cytokine milieu leads to the generation of a Th-17 profile which is associated with an increase in osteoclastogenic activity and MM cell growth ${ }^{25}$. Myeloid-derived suppressor cells (MDSCs) also mediate the suppression of T-cell-mediated immunity against $M M$ cells via interaction with $T_{\text {regs }}$ and also play a role in progression and resistance to therapy in $\mathrm{MM}^{26}$. Additionally, plasmacytoid dendritic cells (DCs), which are important in their role as APCs are often dysfunctional and release various soluble factors like VEGF, IL-6, SDF- $1 \alpha$ among others upon interaction with MM cells, which in turn promote the growth and survival of myeloma cells ${ }^{27}$. Indoleamine 2,3 dioxigenase-1 (IDO-1), an enzyme involved in tryptophan metabolism, is also overexpressed by myeloma cells through the action of HGF, which increases the generation of kynurenines that contribute to the immune suppressive microenvironment in the $\mathrm{BM}^{28}$. IDO- 1 also leads to the expansion of $\mathrm{T}_{\text {regs }}$ further contributing to modulation of $\mathrm{T}$-cell function ${ }^{28}$. Thus, various factors contribute to the development of immune dysregulation in MM, which in turn is thought to play a role in promoting growth, prolonging survival, and conferring resistance to therapy of MM cells.

\section{Immunotherapy in MM: a multifaceted approach}

Newer agents looking at immune targeting of MM cells can be divided into three broad types (Fig. 1).

1. Monoclonal antibodies (mAbs) targeting antigens expressed by MM cells.

2. Treatment strategies to reverse the immune tolerance towards MM cells-the checkpoint inhibitors. 


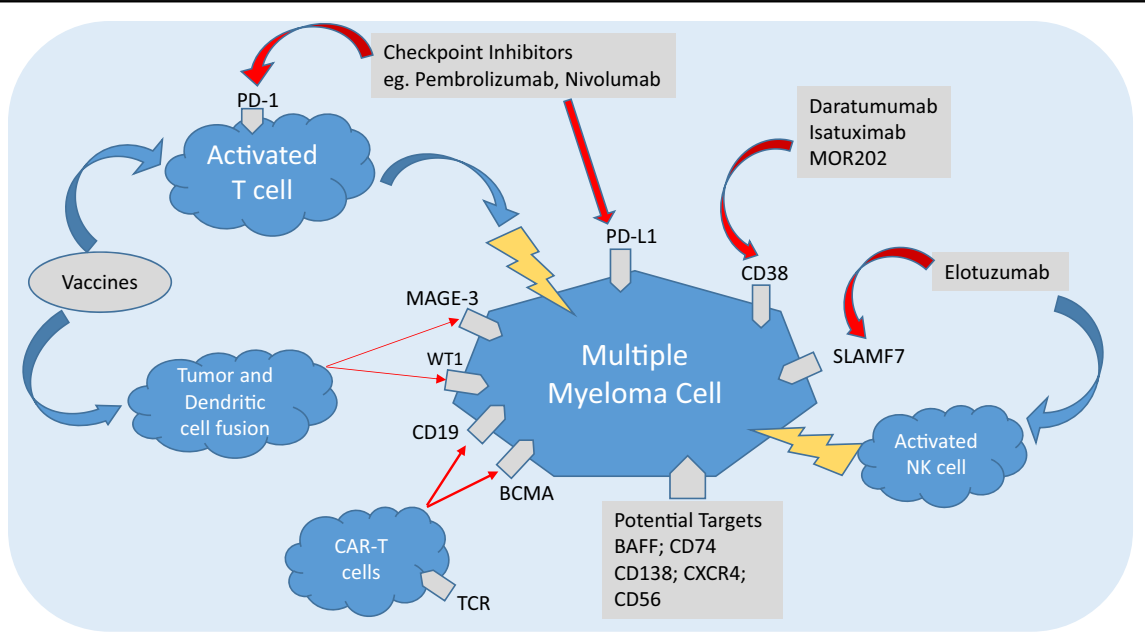

Fig. 1 Mechanisms of action of various immunotherapeutic agents for treating multiple myeloma. BAFF B-cell activating factor, BCMA B-cell maturation antigen, CAR-T cells chimeric antigen receptor T cells, CXCR4 chemokine receptor 4, MAGE-3 melanoma-associated antigen-3, NK cell natural killer cell, PD-1 programmed death receptor-1, PD-L1 programmed death receptor ligand-1, SLAMF7 signaling lymphocyte activating molecule family-7, TCR T-cell receptor, WT1 Wilms tumor 1.

3. Antitumor activity through the generation of higher and more potent immune effector cells against MM (e.g., genetically engineered $\mathrm{T}$ cells, bispecific antibodies, vaccines).

\section{Targets for mAbs in the treatment of MM}

An ideal target antigen for an $\mathrm{mAb}$ would be one with a high and uniform expression on the myeloma cells to have sufficient efficacy and a negligible to low expression on other normal cells to avoid off-target side effects. Various antigens expressed on MM cells have been studied and antibodies against many of these have been developed. Of these, CD38, signaling lymphocyte activating molecule family-7 (SLAMF7), and B-cell maturation antigen (BCMA) represent the most important targets and are discussed in detail.

\section{Physiologic role and clinical efficacy of CD38 targeting}

The CD38 antigen is a cell surface glycoprotein that is involved in signal transduction. Engagement of CD38 stimulates intracellular proliferation signals through a cascade involving various signaling molecules like CD31, ZAP-70, and ERK1/2 ${ }^{29}$. CD38 also acts as an ectoenzyme that promotes the generation of adenosine through the degradation of nicotinamide adenine dinucleotide. Adenosine in turn is thought to promote survival of myeloma cells via modulation of the immune response toward the malignant clone ${ }^{30}$. As a target, CD38 is uniformly expressed on majority of the myeloma cells ${ }^{31}$.

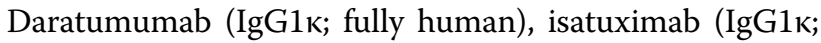
chimeric), and MOR202 (IgG1 $\lambda$; fully human) are mAbs developed and tested against CD38. In preclinical studies, all three antibodies have been shown to cause cell death via antibody-dependent cellular cytotoxicity (ADCC) and complement-dependent cytotoxicity, the classic mechanisms in antibody-mediated cytotoxicity ${ }^{31}$. In addition to these, antibody-dependent cellular phagocytosis and apoptosis also play a role in cell kill by these antibodies. Despite the similarities in mechanism of action, there appear to be some differences in these three antibodies and they bind to different epitopes of CD38. Isatuximab appears to be a more potent inducer of ectoenzyme function of CD38 and unlike daratumumab, leads to apoptosis of MM cells without crosslinking of the Fc receptors of the antibody ${ }^{31}$. However, the clinical implication of these differences is not well established presently.

Daratumumab is the CD38 antibody for which the majority of the clinical trial data currently exists. In the phase II SIRIUS trial, daratumumab monotherapy demonstrated an overall response rate (ORR) of $29 \%$ in a heavily pretreated population that had received a median of five prior lines of therapy ${ }^{32}$. Lenalidomide has shown to upregulate the expression of CD38 on myeloma cells and immunomodulatory drugs (IMiDs) appear to be natural allies to anti-CD38-directed antibodies ${ }^{33}$. The combination of daratumumab with lenalidomide and dexamethasone (Rd) was found to be highly active with an ORR of $81 \%$ and stringent complete response (sCR) rate of $25 \%$ in a pretreated population with a median of 2 prior lines of therapy ${ }^{34}$. Recent phase III clinical trials with daratumumab in combination with both proteasome inhibitors (PIs) and IMiDs have demonstrated a remarkable improvement in the rate of deeper responses including higher minimal residual disease negativity, and improved outcomes in patients with $\mathrm{MM}^{35-37}$. Some of 


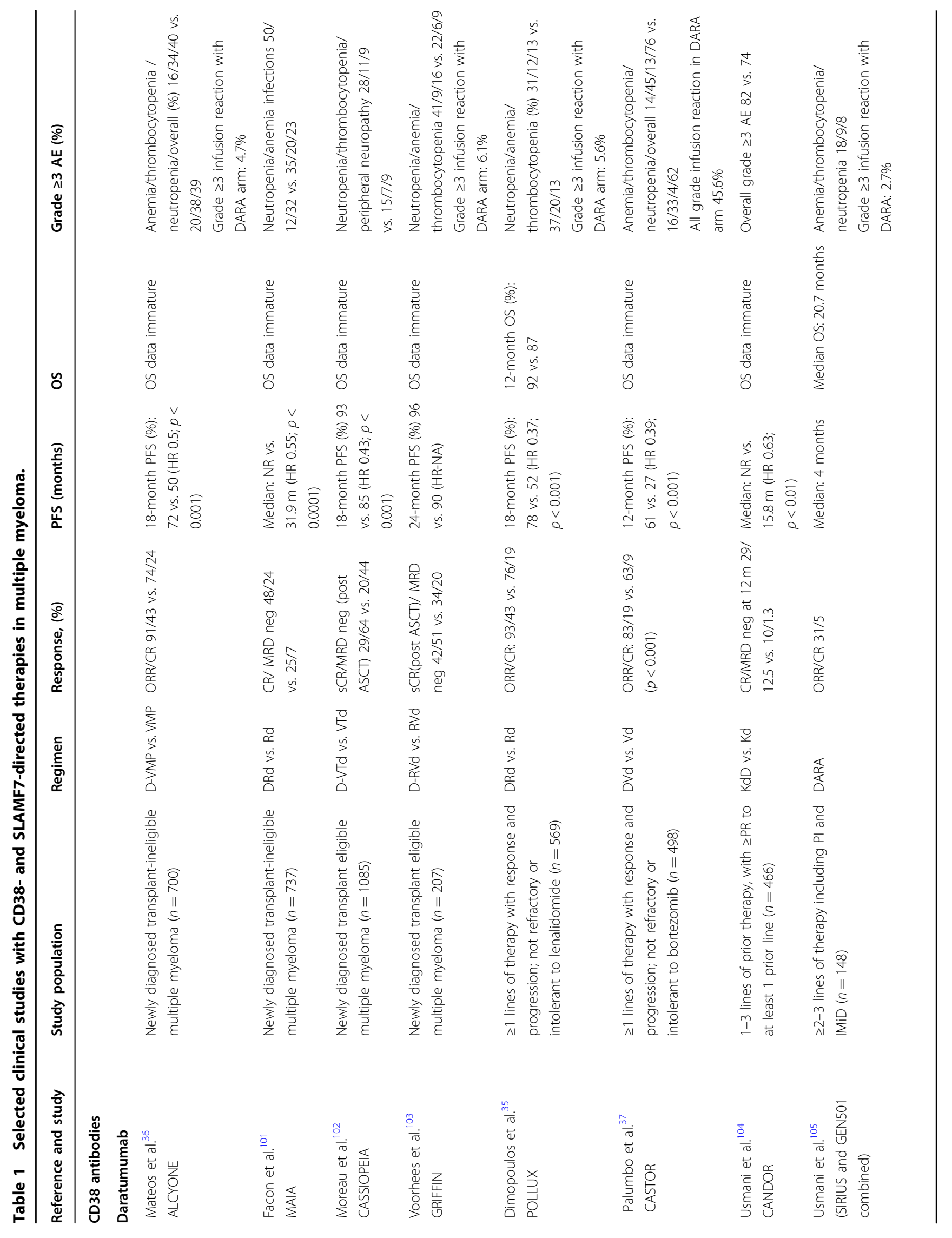




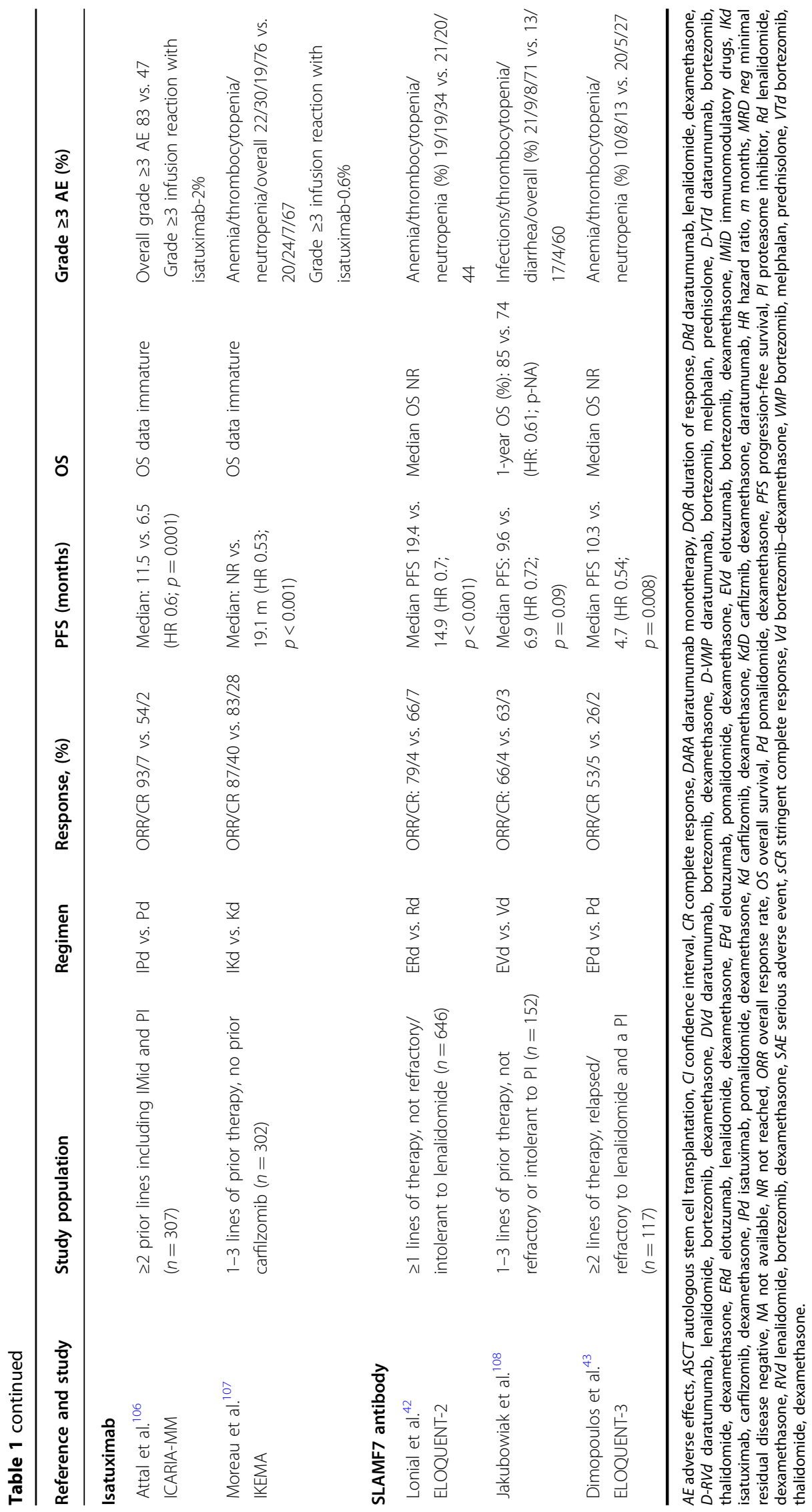


the important clinical trials of CD38-directed mAbs in the frontline and relapsed setting are highlighted in Table 1.

\section{Signaling lymphocyte activating molecule family-7 (SLAMF7)}

This is a cell surface molecule expressed on MM cells as well as on other lymphocytes, including NK cells and functions to modulate the normal immune response and has been shown to promote survival function of myeloma cells $^{38}$. Elotuzumab $(E)$ is a fully humanized $m A b$-directed against SLAMF7. The Fab fragment of elotuzumab binds to the extracellular domain of SLAMF7 and the Fc portion attaches to the CD16 receptor of NK cells ${ }^{39}$. Attachment of elotuzumab to NK cells leads to their activation and degranulation, ultimately causing myeloma cell death by $\mathrm{ADCC}^{40}$. In clinical trials, elotuzumab monotherapy has demonstrated only modest activity, with few patients achieving disease stabilization as the best response $^{41}$. The phase III ELOQUENT-2 trial compared ERd and Rd in 321 patients with previously treated MM (at least 1 prior line of therapy) that were not refractory to lenalidomide $^{42}$. The ORR was significantly better for the elotuzumab arm (79\% vs. $66 \% ; p<0.01)$. At a median follow-up of 24.5 months, the median PFS was 19.4 months for ERd versus 14.9 months for Rd (hazard ratio [HR] 0.7; 95\% confidence interval [CI] 0.57-0.85; $p<$ $0.01)^{42}$. Elotuzumab has also been studied in combination with pomalidomide and dexamethasone (EPd) in a phase 2 study (ELOQUENT-3) for RRMM with at least two prior lines of therapy, including lenalidomide and PI. The primary endpoint of the study was PFS and EPd was associated with a significant improvement in PFS (10.4 vs. 4.7 months; HR 0.54, $p=0.008$ ) compared to pomalidomide and dexamethasone (Table 1) ${ }^{43,44}$. Elotuzumab in combination with lenalidomide, bortezomib, and dexamethasone (RVd) has also been studied by Usmani et al. in a phase 1 study of eight patients with newly diagnosed MM and demonstrated a good safety profile with a comparable toxicity profile to $\mathrm{RVd}^{45}$.

\section{B-cell maturation antigen (BCMA)}

A critical part of immune-based therapy is the selection of an appropriate tumor-specific antigen, which should ideally be uniformly expressed on the MM plasma cells with limited or no expression on the normal cells ${ }^{46}$. This can be challenging, especially due to the development of sub-clones that can impart potential phenotypic differences to the MM cells ${ }^{46}$. A well-suited target appears to be BCMA (CD269), which is a member of the tumor necrosis factor receptor family ${ }^{47}$. Its expression is restricted to some B-cell lineage cells with fairly uniform expression on normal plasma cells and MM cells with almost no expression on non-hematologic cells, making it an ideal target antigen ${ }^{48}$. BCMA-directed therapy represents an important treatment option in heavily pretreated $\mathrm{MM}^{49,50}$. Belantamab mafodotin (GSK2857916) is an antibody drug conjugate (ADC) consisting of an afucosylated anti-BMCA IgG1 mAb conjugated to monomethyl auristin-F (MMAF). The ADC acts by binding to the surface of MM cells expressing BCMA followed by internalization of MMAF which acts by microtubule disruption and inhibition of mitosis. The afucosylated arm also helps to promote ADCC against BCMA expressing cells $^{51}$. Belantamab mafodotin has been evaluated in RRMM who had received prior alkylator therapy, PIs, IMiDs, and stem cell transplant and demonstrated promising activity in phase 1 study with a $60 \%$ ORR in the $3.4 \mathrm{mg} / \mathrm{kg}$ cohort, including one $\mathrm{sCR}$ and two complete responses (CR). The median PFS was 7.9 months $(95 \% \mathrm{CI}$ : 3.1-not reached) ${ }^{51}$. Corneal side effects (blurred vision, dry eye, and photophobia) were common adverse events noted in $63 \%$ of the patients in the $3.4 \mathrm{mg} / \mathrm{kg}$ dose cohort. These corneal side effects were reversible and attributed to the payload (MMAF) used in belantamab mafodotin with similar adverse effects have been consistently described in various other ADCs incorporating $M_{M A F^{52}}$. Grade $\geq 3$ thrombocytopenia and anemia were noted in $34 \%$ and $14 \%$ patients, respectively ${ }^{51}$. The subsequent phase 2 trial (DREAMM-2) studied belandatamab mafodotin in two dose regimens of 2.5 and $3.4 \mathrm{mg} / \mathrm{kg}^{53}$. The median lines of prior therapies were 6 and 7, respectively. Majority of the patients in both the cohorts were refractory to PIs (76\% and $75 \%$ refractory to bortezomib, $65 \%$ and $58 \%$ refractory to carfilzomib), IMiDs (90\% and $89 \%$ refractory to lenalidomide, $87 \%$ and $78 \%$ refractory to pomalidomide), and daratumumab (100\% and $92 \%)$ in the 2.5 and $3.4 \mathrm{mg} / \mathrm{kg}$ dose arms, respectively ${ }^{53}$. At the data cutoff date, the ORR was 31\% (30/97) and 34\% (34/99) in the 2.5 and $3.4 \mathrm{mg} / \mathrm{kg}$ dose arms, respectively. Grade $\geq 3$ keratopathy was noted in $27 \%$ and $21 \%$ in the 2.5 and $3.4 \mathrm{mg} / \mathrm{kg}$ dose arms, respectively, and was the most common reason for permanent discontinuation of therapy. After a median follow-up of 6.5 months, the median PFS was 2.9 months (95\% CI: $2.1-3.7$ ) in the $2.5 \mathrm{mg} / \mathrm{kg}$ cohort and 4.9 months ( $95 \% \mathrm{CI}: 2.3-6.2$ ) in the $3.4 \mathrm{mg} / \mathrm{kg}$ cohort. The OS data were not mature at the time of reporting ${ }^{53}$. Newer ADCs utilizing BCMA as a target, including MEDI2228 (incorporating Pyrrolobenzodiazepine) and HDP-101 (incorporating Amanitin), are also under evaluation ${ }^{50}$.

\section{Antibodies against other targets}

Antibodies have also been developed against other promising antigens in MM. Tabalumab, an $\mathrm{mAb}$ against B-cell activating factor (BAFF), has been tested in a doseescalation phase I study in combination Vd in RRMM. 
Table 2 Additional monoclonal antibodies being studied in the treatment of MM.

\begin{tabular}{|c|c|c|c|}
\hline & Target & $\begin{array}{l}\text { Phase } \\
\text { of study }\end{array}$ & Response \\
\hline \multicolumn{4}{|l|}{ Monoclonal antibody } \\
\hline Tabalumab ${ }^{54}$ & BAFF & $\|$ & $\begin{array}{l}\text { ORR of } 56 \% \text { in RRMM in combination with Vd; a phase ॥ } \\
\text { combination therapy study failed to show improvement in } \\
\text { progression-free survival compared to placebo }\end{array}$ \\
\hline Milatuzumab ${ }^{56}$ & CD74 & $|/| \mid$ & $\begin{array}{l}\text { No OR as monotherapy in RRMM; } 26 \% \text { had SD for }>3 \text { months } \\
\text { (median } 5 \text { lines of therapy) }\end{array}$ \\
\hline Siltuximab $b^{109}$ & IL-6 & $\|$ & $\begin{array}{l}\text { No OR as monotherapy; combination with dexamethasone } \\
\text { showed } 17 \% \text { PR (median } 4 \text { lines of prior therapy) }\end{array}$ \\
\hline \multicolumn{4}{|l|}{ Antibody drug conjugates } \\
\hline Indatuximab ravtansine ${ }^{57}$ & $\begin{array}{l}\text { CD138 (payload: } \\
\text { maytansinoid DM4) }\end{array}$ & I/lla & ORR of $78 \%$ with Rd in RRMM (median 4 lines of prior therapy) \\
\hline $\begin{array}{l}\text { Belanatmab mafadotin } \\
(\text { GSK2857916) }\end{array}$ & BCMA (payload: MMAF) & $\|$ & $\begin{array}{l}\text { ORR: } 31 \%(30 / 97) \text { in } 2.5 \mathrm{mg} / \mathrm{kg} \text { dose and } 34 \%(34 / 99) \text { in the } 3.4 \mathrm{mg} / \\
\mathrm{kg} \text { dose in a heavily pretreated and refractory population }\end{array}$ \\
\hline \multicolumn{4}{|c|}{ Antibodies against immune checkpoints } \\
\hline Pembrolizumab ${ }^{65}$ & PD-L1 & । & $\begin{array}{l}\text { ORR of } 76 \% \text { in combination with Rd (median } 3 \text { lines of prior } \\
\text { therapies) }\end{array}$ \\
\hline Nivolumab 61,66 & PD-1 & । & $\begin{array}{l}\text { Best response of SD in } 63 \% \text { as monotherapy in RRMM }(n=27) \text { with } \\
\text { OR in } 1(4 \%) \text { patient; no objective response noted with } \\
\text { combination of nivolumab with ipilimumab in the subset of RRMM } \\
(n=7)\end{array}$ \\
\hline Atezolizumab $^{67}$ & PD-L1 & $\mathrm{lb}$ & $\begin{array}{l}\text { VGPR or better with atezolizumab combination therapy: } 67 \%(4 / 6) \\
\text { in combination with daratumumab and pomalidomide } 44 \%(3 / 7) \\
\text { with lenalidomide and daratumumab 50\% (3/6) with } \\
\text { daratumumab (1-3 prior lines of therapy) }\end{array}$ \\
\hline
\end{tabular}

$B A F F$ B-cell activating factor, $B C M A$ B-cell maturation antigen, $M M A F$ monomethyl auristatin $\mathrm{F}$, $O R$ objective response, $O R R$ overall response rate, $P R$ partial response, $R d$ lenalidomide-dexamethasone, RRMM relapsed refractory multiple myeloma, SD stable disease, Vd bortezomib-dexamethasone.

The combination was well tolerated with an ORR of $56 \%{ }^{54}$. However, a phase II study of tabalumab 100 or $300 \mathrm{mg}$ in combination with $\mathrm{Vd}$ failed to show an improvement in the PFS of patients with tabalumab and Vd compared to the placebo-Vd $\operatorname{arm}^{55}$. Milatuzumab is a humanized anti-CD74 antibody that has demonstrated disease stabilization in a heavily pretreated population of MM when used as monotherapy but objective responses were lacking ${ }^{56}$. Indatuximab ravtansine, an ADC comprising of an mAb against CD138 conjugated with maytansinoid DM4 (microtubule-binding cytotoxic agent), demonstrated only modest clinical activity as monotherapy in RRMM, but was found to be fairly well tolerated in combination with dexamethasone and lenalidomide or pomalidomide, with a median duration of response of 21 months and ORR of $77 \%$ in evaluable patients $^{57}$. Antibodies against many other targets, including IL- 6 and CXCR4, have also been developed and are in early phases of testing (Table 2$)^{58}$.

\section{Strategies to reverse the immune tolerance towards MM cells: PD-1/PD-L1-directed therapy in MM}

The PD-1/PD-L1 axis is a negative co-stimulatory pathway that plays a crucial role in regulating immune response. While physiologically it is important to prevent autoimmunity, its overexpression leads to immune evasion and development of tolerance against tumor cells in various malignancies ${ }^{10}$. Plasma cells in patients with MM have demonstrated to have increased PD-L1 expression ${ }^{12}$. Similarly, the circulating $\mathrm{T}$ cells and NK cells in patients with MM demonstrate increased expression of PD-1 receptor $^{59}$. The binding of PD-L1 on myeloma cells to the PD-1 receptor on NK cells and T cells leads to a decline in the Th-1 cytokines resulting in T-cell apoptosis and attenuation of T-cell immune effector functions ${ }^{60}$. Despite a strong rationale for the role of immune checkpoint inhibitors in MM, no objective response was noted in the phase I study of pembrolizumab (PD-L1 
antibody) monotherapy for RRMM, with the best response achieved being disease stabilization ${ }^{61}$. In a phase I study looking at efficacy of nivolumab in hematologic malignancies, the best response with nivolumab monotherapy in the subset of patients with RRMM $(n=27)$ was stable disease $(63 \%)$ with objective response noted in only one patient $(4 \%)^{61}$.

Lenalidomide and pomalidomide appear to enhance sensitivity of checkpoint inhibitors in MM and a combination of these regimens can inhibit the proliferative effects of the BMSCs on myeloma cells and reverse the immunoparesis due to the MDSCs ${ }^{62}$. In a phase I doseescalation study, the combination of lenalidomide and dexamethasone with the PD-L1 antibody pembrolizumab demonstrated an ORR of $76 \%$ with responses noted in patients that were refractory to lenalidomide as well ${ }^{63}$. Pembrolizumab has also been studied in combination with pomalidomide and dexamethasone (KEYNOTE-183) in a predominantly PI and lenalidomide refractory population $^{64}$. The combination demonstrated an ORR of $60 \%$ in the double-refractory subset; the common grade $\geq 3$ hematologic toxicities included neutropenia (40\%) and anemia (21\%), whereas interstitial pneumonitis was noted in $15 \%$ patients and was predominantly low grade ${ }^{64}$. The KEYNOTE-185 is a phase III study comparing pembrolizumab $+R d$ and $R d$ in patients with newly diagnosed transplant-ineligible $\mathrm{MM}^{65}$. Preliminary data after recruitment of 301 patients out of the target of 640 patients suggest higher rates of toxicity, especially immune-mediated toxicities (including hyper/hypothyroidism, colitis, skin reactions) in the pembrolizumab $\mathrm{arm}^{65}$. In view of these adverse signals, the FDA had put the KEYNOTE-183 and KEYNOTE-185 studies on hold at the time of this write-up.

Nivolumab was also studied in combination with ipilimumab but the combination did not demonstrate any objective response in the subset of RRMM $(n=5)^{66}$. A phase $1 \mathrm{~b}$ clinical trial presented its abstract form (NCT02431208) studied atezolizumab in combination with daratumumab $(n=11)$ with or without addition of lenalidomide $(n=7)$ or pomalidomide $(n=6)^{67}$. A very good partial response (VGPR) or better was observed in $50 \%(3 / 6)$ patients treated with atezolizumab with daratumumab with $1-3$ prior lines of therapy, $43 \%(3 / 7)$ patients treated with atezolizumab, daratumumab, lenalidomide and $67 \%(4 / 6)$ patients treated with atezolizumab, daratumumab, pomalidomide. The sample size was small to draw reliable conclusions and were no new safety signals reported ${ }^{67}$. Concerns seen in the KEYNOTE-183 and KEYNOTE-185 studies also resulted in complete or partial hold on vast majority of clinical trials of combinations of various checkpoint inhibitors (nivolumab, durvalumab, atezolizumab, etc.) with IMiDs at the time of this write-up. Phase II study of nivolumab and lenalidomide (NCT03333746) in RRMM and phase I study of nivolumab-pomalidomide-dexamethasone \pm elotuzumab (NCT03023527) in RRMM have been terminated. Similarly, studies of nivolumab combinations (NCT02903381) and atezolizumab monotherapy (NCT02784483) in asymptomatic/smoldering MM have been either suspended or terminated. The most up to date details of the study status of PD-1/PD-L1 inhibitors in $\mathrm{MM}$ can be found at www.clinicaltrials.gov.

\section{Enhancing the generation of myeloma-specific $T$ cells and immune effector cells Chimeric antigen receptor-T cell (CAR-T) therapy}

CAR-T cell therapy is a form of adoptive cellular therapy wherein the host's $T$ cells are genetically engineered to express tumor-specific antigens. Chimeric antigen receptors consist of antigen recognition domains and $\mathrm{T}$-cell signaling moieties that are expressed on T-cells cultured from the patient through the use of vectors ${ }^{68}$. CAR-T cells can selectively target the antigens based on the antigen recognition domains expressed on these cells, thus imparting specificity to the $\mathrm{T}$-cell responses resulting in a more effective tumor cell kill with decreased off-target effects, all without any human leukocyte antigen restriction ${ }^{69}$. The first-generation CAR-T cell therapies lacked a costimulatory domain and were associated with only modest responses ${ }^{70}$. However, the second-generation CAR-T cell therapies incorporated co-stimulatory domains, commonly CD28 or $4-1 \mathrm{BB}$, and have shown substantial improvement in the efficacy of these CAR-T cells ${ }^{71}$. The generation of CAR-T cells is followed by administration of a conditioning chemotherapy to the patient, typically cyclophosphamide or combination of cyclophosphamide with fludarabine, followed by reinfusion of the CAR-T cells into the patient ${ }^{69}$.

The first-in-human clinical trial with BCMA-directed CAR-T cells in a heavily pretreated population (median of seven prior lines of therapy) demonstrated that the efficacy and toxicity of CAR-T therapy were dose dependent $^{72}$. In the two patients treated at the highest dose of CAR-T $\left(9 \times 10^{6}\right.$ CAR-T cells $\left./ \mathrm{kg}\right)$, one patient achieved an sCR lasting for 17 weeks and another patient achieved a VGPR 28 weeks after the infusion of CAR-T cells; the responses were lower in patients treated at lower doses, with only one of the ten patients going on to achieve a $\mathrm{VGPR}^{72}$. The toxicity profile was similar to that seen with CAR-T cell therapy in patients with acute leukemia, with cytokine release syndrome (CRS) being the most noticeable unique toxicity ${ }^{72}$. CRS is a unique toxicity noted with therapies acting via $\mathrm{T}$-cell proliferation strategies (CAR- $\mathrm{T}$ and bispecific antibodies) and represents a systemic inflammatory response that can present with varied clinical manifestations including fever, fatigue, arthralgias, rash, and hypotension. The initial lack of awareness regarding the manifestations of CRS led to unfavorable 


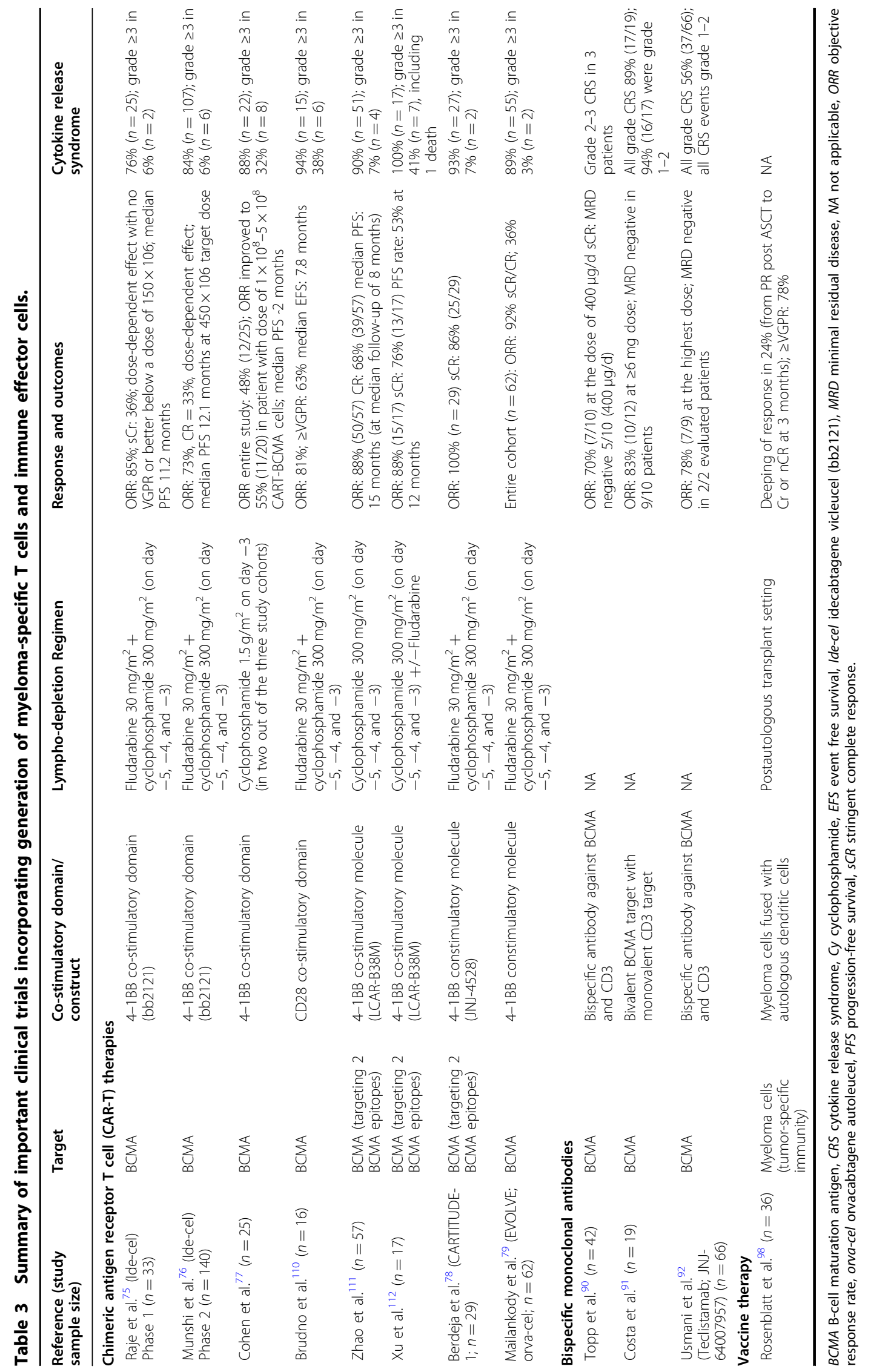


outcomes including mortalities in early clinical trials with CAR-T therapy. Subsequently, the improved understanding of CRS and increased vigilance for the same has helped in earlier institution of treatment with corticosteroids and the anti-IL6 antibody tocilizumab for $\mathrm{CRS}^{73}$. Neurological toxicity is also common with CAR-T therapy and can present with a broad variety of symptoms ranging from confusion to the more severe ones in the form of aphasia and encephalopathy ${ }^{74}$.

Idecabtagene vicleucel (ide-cel, bb2121) is a secondgeneration BCMA-directed CAR-T cell therapy that incorporates $4-1 \mathrm{BB}$ as a co-stimulatory domain and has shown promising results ${ }^{75}$. The phase I dose-escalation study included patients with heavily pretreated MM patients with $\geq 50 \%$ BCMA expression and a measurable disease. The generation of the CAR-T cells was successful in all patients. The patients received $50 \times 10^{6}, 150 \times 10^{6}$, $450 \times 10^{6}$, or $800 \times 10^{6}$ CAR-T cells in the dose-escalation phase and $150 \times 10^{6}-450 \times 10^{6}$ in the expansion phase of the study ${ }^{75}$. In the 33 evaluable patients, the ORR was $85 \%$ with $15(45 \%)$ patients achieving a CR. Notably, the ORR was an impressive $74 \%$ in patients with high-risk cytogenetics in this trial. The rate of CRS was $76 \%$ with grade $\geq 3$ CRS seen in 6\% patients. Neurologic toxicities were noted on $42 \%$ patients, with a vast majority being grade 1 or 2 . After a median follow-up of 11 months, the median PFS with ide-cel was an impressive 11.8 months (95\% CI: 6.2-17.8 months $)^{75}$. Early phase 2 results of ide-cel (KarMMa) were recently presented at the American Society of Clinical Oncology 2020 meeting $^{76}$. The majority of the included patients were refractory to PIs, IMiDs, and CD38-directed therapy. Of the 140 patients that had undergone leukapheresis, 128 had received idecel infusion at the time of reporting. The success rate for manufacturing of ide-cel was $99 \%$ and patients were treated in doses of $150 \times 106(n=4), 300 \times 106(n=70)$, and $450 \times 106(n=56)$. Peak CAR-T population was noted at day 11 of infusion and the ORR and CR for the entire cohort was $73 \%$ and $33 \%$, respectively which met the primary endpoint of the study. Higher response rates were noted with escalating doses (ORR/CR of $50 \% / 25 \%$, $69 \% / 29 \%$, and $82 \% / 39 \%$ in $150 \times 106,300 \times 106$, and $450 \times 106$ dose cohorts, respectively) ${ }^{76}$. The efficacy was maintained across all major subgroups. After a median follow-up of 13.3 months, the median PFS also demonstrated a target dose-based increment with median PFS of $2.8,5.8$, and 12.1 months at dose of $150 \times 106,300 \times 106$, and $450 \times 106$, respectively. The PFS improved with the depth of response with a median PFS of 20 months in patients achieving $\geq \mathrm{CR}$. The OS data for ide-cel was not mature at the time of the presentation. The rate of CRS was higher with increased target dose $(50 \%, 76 \%$, and $96 \%$ in $150 \times 106,300 \times 106$, and $450 \times 106$ target dose cohorts, respectively). Overall, CRS was predominantly low grade with only $6 \%$ patients developing grade $\geq 3$ CRS (one patient had grade $5 \mathrm{CRS})^{76}$.

Phase I study results for another BCMA-directed CAR-T therapy with 4-1BB co-stimulatory signaling domain have been reported recently ${ }^{77}$. There was no pre-specified BCMA expression level required for inclusion in the study. The 25 patients included in the study were treated in three cohorts: BCMA CAR-T cells alone at a dose of $1-5 \times 108$ (cohort 1 ), $1.5 \mathrm{~g} / \mathrm{m}^{2}$ of cyclophosphamide with $1-5 \times 107$ BCMA CAR-T cells (cohort 2), and the third group with $1.5 \mathrm{~g} / \mathrm{m}^{2}$ of cyclophosphamide with $1-5 \times 108$ BCMA CAR-T cells (cohort 3 ). The response rates with therapy were $44 \%$ in cohort $1,20 \%$ in cohort 2 and $64 \%$ in cohort 3 . The rate of CRS was $88 \%$ with grade $3-4$ CRS noted in $32 \%$ patients. Grade $3-4$ neurological toxicity was noted in three (12\%) patients ${ }^{77}$. Another promising CAR-T construct is JNJ-4528 that targets CD3 and two epitopes of BCMA. Efficacy and safety profile appears to be excellent and updated results of the CARTITUDE- 1 study demonstrate an ORR of $100 \%$ with $86 \%$ achieving an $\mathrm{sCR}^{78}$. Orvacabtagene autoleucel (orva-cel) is another promising BCMA-directed CAR-T therapy with a low affinity for soluble BCMA that has demonstrated an excellent manufacturing success rate (100\% at the time of reporting) with low rates of grade $\geq 3$ CRS ( $3 \%$ for the entire cohort) and excellent efficacy profile with $\mathrm{CR} / \mathrm{sCR}$ rates of $36 \%$ for the entire cohort in the recently reported results of the phase 1/2 EVOLVE study ${ }^{79}$. The BCMA-directed CAR-T cell therapy appears to be a very promising treatment strategy as we await further clinical trial data with a longer follow-up. Important CAR-T therapy studies are summarized in Table 3. Development of CAR-T therapy against CD38 and CD138, both expressed uniformly on MM cells, has been successful in preclinical setting and likely to add to this rapidly expanding field ${ }^{80,81}$.

There are some hindrances with the use of CAR-T cell therapy in MM. The cells may lose the tumor-specific antigen over time leading to loss of response as was seen in first-generation CARs ${ }^{46}$. The incorporation of a second co-stimulatory molecule in the structure of the CAR-T cells, in addition to the $\mathrm{CD} 3 \zeta \mathrm{T}$-cell activation domain, helps to generate a more effective and long-lasting immune response as has been seen in the more recent clinical trials ${ }^{76,82}$. Nonetheless, most patients still relapse after CAR-T therapy. Also, the tumor microenvironment in $M M$ is immune suppressive and may hamper an adequate $\mathrm{T}$-cell response after infusion of these cells ${ }^{8,23}$. Further alterations in the CAR structure like targeting multiple antigens to account for multiple clones may help to overcome some of the limitations ${ }^{83}$.

\section{Bispecific monoclonal antibodies (BsMAb)}

A bispecific monoclonal antibody (BsMAb) concomitantly binds to two different antigens, commonly one 
being on $\mathrm{T}$ cells and the other being a tumor-associated antigen, thereby redirecting the cytotoxic $\mathrm{T}$ cells toward the tumor cells. The BsMAb therapy is similar in its mechanism to CAR-T therapy in the sense that both rely on host's $T$ cells to elicit antitumor response. However, BsMAb comes with the obvious advantage of not requiring a processing period that is associated with the generation of CAR-T cells and hence have earned the appellation of off-the-shelf CAR-T therapy ${ }^{84}$. Various bispecific antibody platforms are currently in clinical trials. The BiTE $^{\circledR}$ (bispecific T-cell engager; Amgen, Thousand Oaks, CA, USA) platform for BsMAb consists of two single-chain variable fragments ( $\mathrm{scFv}$ ), one binding to $\mathrm{CD} 3$ on $\mathrm{T}$ cells and the other to a tumor-associated antigen $^{85}$. DuoBody ${ }^{\circledR}$ (Genmab A/S, Copenhagen, Denmark) is another bispecific antibody platform that consists of heavy and light chain homodimers from two different antibodies that fused together with a controlled Fab-arm exchange at matched point $\mathrm{CH}-3$ mutations to generate a single monoclonal IgG1 heterodimer. The potential advantage of this platform is that it is stable and retains the functional and structural integrity of native IgG molecule ${ }^{86,87}$. Another platform in clinical trials is DualAffinity Re-Targeting (DART ${ }^{\circledR}$, Macrogenics Inc.) which incorporates c-peptide disulfide linkages to further stabilize the two different diabodies ${ }^{88}$. Currently, the majority of clinical trial data for BsMAbs in MM is limited to the $\mathrm{BiTE}^{\circledR}$ platform.

There is good preclinical evidence of selective lysis of BCMA positive myeloma cells in ex vivo assays by bispecific antibody targeting $\mathrm{BCMA} / \mathrm{CD}^{89}$. A first-inhuman study of the BCMA-directed BiTE ${ }^{\circledR}$ AMG420 comprised of 6-week cycles of the experimental drug given for 5 or less cycles depending upon toxicity and progression. Of the 42 heavily pretreated patients enrolled in the trial, 13 (31\%) had an objective response. At the tolerated dose of $400 \mu \mathrm{g} /$ day dose, seven out of ten patients responded. More importantly, five out of these seven patients achieved an $\mathrm{sCR}^{90}$. Three patients developed grade 2-3 CRS and treatment-related adverse effect associated mortality was noted in two patients (adenoviral infection associated fulminant hepatitis and pulmonary

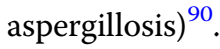

Another newer BsMAb is CC-93269 which binds bivalently to BCMA and monovalently to $\mathrm{CD} 3 \varepsilon$ in a 2:1 fashion $^{91}$. In a phase I study of 19 patients with RRMM with $\geq 3$ previous lines of therapy (BCMA-therapy naive), CC-93269 was studied in doses ranging from 0.15 to $10 \mathrm{mg}^{91}$. All patients were heavily pretreated (median 6 lines of therapy) with a vast majority of patients being refractory to daratumumab as well as last PI and/or IMiD. At doses $\geq 6 \mathrm{mg}$ in cycle 1, CC-93269 demonstrated remarkable activity with 10 out of 12 (83\%) patients achieving a $\geq \mathrm{PR}$ and $58 \%$ achieving $\geq V G P R$. In the ten patients that underwent MRD testing, nine of these achieved MRD negativity ${ }^{91}$. The toxicity profile was noticeable for CRS in 17 out of the 19 patients (89\%), which was predominantly grade 1-2 (in 16/ 17 patients with CRS), while one of the 17 patients being treated with $10 \mathrm{mg}$ on day 8 died in the setting of CRS. Cytopenias and infections represented the other common grade $\geq 3$ adverse effects. Data for PFS and OS were not mature at the time of this write-up ${ }^{91}$.

A recently reported phase 1 study of Teclistamab (JNJ64007957), a bispecific BCMA/CD3 antibody, demonstrated a good tolerability and safety profile with $7 / 9$ (78\%) patients achieving a response at the highest dose ${ }^{92}$. Another BCMA/Cd3 bispecific antibody under initial phases of in-human testing is REGN5458. Preliminary results of three patients treated at a starting dose of $3 \mathrm{mg}$ revealed responses in two patients with disease progression the third patient at first assessment. No doselimiting toxicities were noted and additional data are awaited $^{93}$.

\section{Vaccine therapy for MM}

Strategies for developing vaccines against MM have been around for more than two decades now. Most of the initial strategies involved noncellular approaches employing tumor antigens for inducing immune response. The most commonly employed antigen was the idiotypic antigen that forms the variable fragment of the monoclonal immunoglobulin in $\mathrm{MM}^{5}$. Idiotypic antigen-based vaccines had demonstrated poor immunogenicity and have been supplanted by more specific tumor antigens like Cancer Testis Antigens MAGE-3 and NY-ESO-1, WT1, and CS1 amongst others ${ }^{94}$. Tumor cell lysates and apoptotic tumor cells have also been used as the source of antigen for the generation of vaccines in order to achieve better immunogenicity, but clinical benefit with these strategies remained elusive ${ }^{95}$.

A promising approach appears to be the use of fusion vaccines that employ autologous DCs fused with tumor cells. DCs are potent APCs and this fusion strategy aims at harnessing the ability of DCs to present multiple tumor antigens to the host $\mathrm{T}$ cells ${ }^{96}$. Phase I studies demonstrated this vaccine to be safe and efficacious ${ }^{97}$. In a subsequent phase II study $(n=36)$, the administration of this vaccine in the postautologous stem cell transplant setting demonstrated a doubling of the myeloma-specific $\mathrm{CD} 4+$ and $\mathrm{CD} 8+\mathrm{T}$ cells ${ }^{98}$. Seventy-eight percent patients achieved a $\geq$ VGPR with this strategy. Notably, $24 \%$ patients who achieved only a PR postautologous transplant converted to a CR or near CR with the use of the fusion vaccine. However, loss of response over time seems to be present ${ }^{98}$. Combining IMiDs or checkpoint inhibitors with these vaccines may have some value in improving both the duration as well as amplitude of response to vaccine therapy ${ }^{99,100}$. 


\section{Conclusions}

Immune dysregulation plays a key role in the pathogenesis and disease progression of MM and restoration of a robust immune response toward the myeloma clone represents an important therapeutic strategy. The rapid evolution of CAR-T cell therapy and BiTEs has already started to reshape the treatment of RRMM.

\section{Conflict of interest}

S.K. -research funding for clinical trials to the institution: Celgene, Takeda, Janssen, BMS, KITE, Merck, Abbvie, Medimmune, Novartis, Roche-Genentech, Amgen, Tenebio, Carsgen; Consulting/Advisory Board participation (with no personal payments): Celgene, Takeda, Janssen, Abbvie, Genentech, Amgen, Molecular Partners and (with personal payment) Oncopeptides, Genecentrix, Cellectar. The remaining authors have no conflicts of interest to disclose.

\section{Publisher's note}

Springer Nature remains neutral with regard to jurisdictional claims in published maps and institutional affiliations.

Received: 19 June 2020 Revised: 24 July 2020 Accepted: 27 July 2020 Published online: 22 August 2020

\section{References}

1. Palumbo, A. \& Anderson, K. Multiple myeloma. N. Engl. J. Med. 364 1046-1060 (2011).

2. Kumar, S. K. et al. Continued improvement in survival in multiple myeloma: changes in early mortality and outcomes in older patients. Leukemia $\mathbf{2 8}$ 1122-1128 (2014).

3. Zanwar, S., Abeykoon, J. P. \& Kapoor, P. Challenges and strategies in the management of multiple myeloma in the elderly population. Curr. Hematol. Malig. Rep. 14, 70-82 (2019).

4. Kumar, S. K. et al. NCCN guidelines insights: multiple myeloma, Version 1.2020. J. Natl. Compr. Canc. Netw. 17, 1154-1165 (2019).

5. Hoyos, V. \& Borrello, I. The immunotherapy era of myeloma: monoclonal antibodies, vaccines, and adoptive T-cell therapies. Blood 128, 1679-1687 (2016).

6. Donato, M. L. et al. The graft-versus-myeloma effect: chronic graft-versus-host disease but not acute graft-versus-host disease prolongs survival in patients with multiple myeloma receiving allogeneic transplantation. Biol. Blood Marrow Transplant. 20, 1211-1216 (2014).

7. Kumar, S. et al. Trends in allogeneic stem cell transplantation for multiple myeloma: a CIBMTR analysis. Blood 118, 1979-1988 (2011).

8. Romano, A. et al. Immunological dysregulation in multiple myeloma microenvironment. Biomed. Res. Int. 2014, 198539 (2014).

9. Mayes, P. A., Hance, K. W. \& Hoos, A. The promise and challenges of immune agonist antibody development in cancer. Nat. Rev. Drug. Discov. 17, 509 (2018).

10. Pardoll, D. M. The blockade of immune checkpoints in cancer immunotherapy. Nat. Rev. Cancer 12, 252-264 (2012).

11. Zou, W. \& Chen, L. Inhibitory B7-family molecules in the tumour microenvironment. Nat. Rev. Immunol. 8, 467 (2008).

12. Liu, J. et al. Plasma cells from multiple myeloma patients express B7$\mathrm{H} 1$ (PD-L1) and increase expression after stimulation with IFN- $\gamma$ and TLR ligands via a MyD88-, TRAF6-, and MEK-dependent pathway. Blood 110, 296-304 (2007).

13. Braga, W. M. T. et al. FOXP3 and CTLA4 overexpression in multiple myeloma bone marrow as a sign of accumulation of $\mathrm{CD} 4(+) \mathrm{T}$ regulatory cells. Cancer Immunol. Immunother. 63, 1189-1197 (2014).

14. Triebel, F. et al. LAG-3, a novel lymphocyte activation gene closely related to CD4. J. Exp. Med. 171, 1393-1405 (1990).

15. Okazaki, T. et al. PD-1 and LAG-3 inhibitory co-receptors act synergistically to prevent autoimmunity in mice. J. Exp. Med. 208, 395-407 (2011).

16. Mussetti, A. et al. PD-L1, LAG3, and HLA-DR are increasingly expressed during smoldering myeloma progression. Ann. Hematol. 98, 1713-1720 (2019).
17. Lucas, F. et al. T cell transcriptional profiling and immunophenotyping uncover LAG3 as a potential significant target of immune modulation in multiple myeloma. Biol. Blood Marrow Transplant. 26, 7-15 (2020).

18. Costa, F., Das, R., Kini Bailur, J., Dhodapkar, K. \& Dhodapkar, M. V. Checkpoint inhibition in myeloma: opportunities and challenges. Front. Immunol. 9, 2204-2204 (2018)

19. Sakaguchi, S. Regulatory T cells: key controllers of immunologic self-tolerance. Cell 101, 455-458 (2000).

20. Sakaguchi, S., Wing, K., Onishi, Y., Prieto-Martin, P. \& Yamaguchi, T. Regulatory T cells: how do they suppress immune responses? Int. Immunol. 21 1105-1111 (2009).

21. Muthu Raja, K. R. et al. Increased T regulatory cells are associated with adverse clinical features and predict progression in multiple myeloma. PLOS ONE $\mathbf{7}$, e47077 (2012)

22. Rutella, S. \& Locatelli, F. Targeting multiple-myeloma-induced immune dysfunction to improve immunotherapy outcomes. J. Immunol. Res. 2012, e196063 (2012).

23. Pratt, G., Goodyear, O. \& Moss, P. Immunodeficiency and immunotherapy in multiple myeloma. Br. J. Haematol. 138, 563-579 (2007).

24. Brocke-Heidrich, K. et al. Interleukin-6-dependent gene expression profiles in multiple myeloma INA-6 cells reveal a $\mathrm{BCl}-2$ family-independent survival pathway closely associated with Stat3 activation. Blood 103, 242-251 (2004).

25. Noonan, K. \& Borrello, I. The immune microenvironment of myeloma. Cancer Microenviron. 4, 313-323 (2011).

26. Malek, E. et al. Myeloid-derived suppressor cells: the green light for myeloma immune escape. Blood Rev. 30, 341-348 (2016).

27. Ratta, M. et al. Dendritic cells are functionally defective in multiple myeloma: the role of interleukin-6. Blood 100, 230-237 (2002).

28. Bonanno, G. et al. Indoleamine 2,3-dioxygenase 1 (IDO1) activity correlates with immune system abnormalities in multiple myeloma. J. Transl. Med. 10 247 (2012)

29. Lee, H. C. Structure and enzymatic functions of human CD38. Mol. Med. 12 317-323 (2006).

30. Horenstein, A. L. et al. A CD38/CD203a/CD73 ectoenzymatic pathway independent of CD39 drives a novel adenosinergic loop in human T lymphocytes. Oncoimmunology 2, e26246 (2013).

31. Donk, N. W. C. Jvd et al. Clinical efficacy and management of monoclonal antibodies targeting CD38 and SLAMF7 in multiple myeloma. Blood 127, 681-695 (2016).

32. Lonial, S. et al. Daratumumab monotherapy in patients with treatmentrefractory multiple myeloma (SIRIUS): an open-label, randomised, phase 2 trial. Lancet 387, 1551-1560 (2016).

33. van der Veer, M. S. et al. Towards effective immunotherapy of myeloma: enhanced elimination of myeloma cells by combination of lenalidomide with the human CD38 monoclonal antibody daratumumab. Haematologica 96, 284-290 (2011).

34. Plesner, $T$. et al. Phase $1 / 2$ study of daratumumab, lenalidomide, and dexamethasone for relapsed multiple myeloma. Blood 128, 1821-1828 (2016).

35. Dimopoulos, M. A. et al. Daratumumab, lenalidomide, and dexamethasone for multiple myeloma. N. Engl. J. Med. 375, 1319-1331 (2016).

36. Mateos, M.-V. et al. Daratumumab plus bortezomib, melphalan, and prednisone for untreated myeloma. N. Engl. J. Med. 378, 518-528 (2018).

37. Palumbo, A. et al. Daratumumab, bortezomib, and dexamethasone for multiple myeloma. N. Engl. J. Med. 375, 754-766 (2016).

38. Hofmeister, C. C. \& Lonial, S. How to integrate elotuzumab and daratumumab into therapy for multiple myeloma. J. Clin. Oncol. 34, 4421-4430 (2016).

39. Richardson, P. G., Lonial, S., Jakubowiak, A. J., Harousseau, J. L. \& Anderson, K. C. Monoclonal antibodies in the treatment of multiple myeloma. Br. J. Haematol. 154, 745-754 (2011).

40. Collins, S. M. et al. Elotuzumab directly enhances NK cell cytotoxicity against myeloma via CS1 ligation: evidence for augmented NK cell function complementing ADCC. Cancer Immunol. Immunother. 62, 1841-1849 (2013).

41. Zonder, J. A. et al. A phase 1, multicenter, open-label, dose escalation study of elotuzumab in patients with advanced multiple myeloma. Blood 120, 552-559 (2012).

42. Lonial, $\mathrm{S}$. et al. Elotuzumab therapy for relapsed or refractory multiple myeloma. N. Engl. J. Med. 373, 621-631 (2015).

43. Dimopoulos, M. A. et al. Elotuzumab plus pomalidomide and dexamethasone for multiple myeloma. N. Engl. J. Med. 379, 1811-1822 (2018). 
44. Dimopoulos, M. A. et al. Elotuzumab plus pomalidomide and dexamethasone for relapsed/refractory multiple myeloma: efficacy results after additional follow-up of the phase 2, randomizaed ELOQUENT-3 study. HemaSphere 3(Suppl 1), 626-627 (2019).

45. Usmani, S. Z. et al. Phase I safety data of lenalidomide, bortezomib, dexamethasone, and elotuzumab as induction therapy for newly diagnosed symptomatic multiple myeloma: SWOG S1211. Blood Cancer J. 5, e334-e334 (2015).

46. Sadelain, M., Brentjens, R. \& Riviere, I. The basic principles of chimeric antigen receptor design. Cancer Discov. 3, 388-398 (2013)

47. Tai, Y. T. \& Anderson, K. C. Targeting B-cell maturation antigen in multiple myeloma. Immunotherapy 7, 1187-1199 (2015)

48. Carpenter, R. O. et al. B-cell maturation antigen is a promising target for adoptive T-cell therapy of multiple myeloma. Clin. Cancer Res. 19, 2048-2060 (2013).

49. Lee, L. et al. Evaluation of B cell maturation antigen as a target for antibody drug conjugate mediated cytotoxicity in multiple myeloma. Br. J. Haematol. 174, 911-922 (2016)

50. Cho, S.-F., Anderson, K. C. \& Tai, Y.-T. Targeting B cell maturation antigen $(B C M A)$ in multiple myeloma: potential uses of BCMA-based immunotherapy. Front. Immunol. 9, 1-15 (2018).

51. Trudel, S. et al. Targeting B-cell maturation antigen with GSK2857916 antibody-drug conjugate in relapsed or refractory multiple myeloma (BMA117159): a dose escalation and expansion phase 1 trial. Lancet Oncol. 19, 1641-1653 (2018)

52. Eaton, J. S., Miller, P. E., Mannis, M. J. \& Murphy, C. J. Ocular adverse events associated with antibody-drug conjugates in human clinical trials. J. Ocul. Pharmacol. Ther. 31, 589-604 (2015).

53. Lonial, S. et al. Belantamab mafodotin for relapsed or refractory multiple myeloma (DREAMM-2): a two-arm, randomised, open-label, phase 2 study. Lancet Oncol. 21, 207-221 (2020).

54. lida, S. et al. Dose-escalation study of tabalumab with bortezomib and dexamethasone in Japanese patients with multiple myeloma. Cancer Sci. 107, 1281-1289 (2016)

55. Raje, N. S. et al. Phase 2 study of tabalumab, a human anti-B-cell activating factor antibody, with bortezomib and dexamethasone in patients with previously treated multiple myeloma. Br. J. Haematol. 176, 783-795 (2017).

56. Kaufman, J. L. et al. Phase I, multicentre, dose-escalation trial of monotherapy with milatuzumab (humanized anti-CD74 monoclonal antibody) in relapsed or refractory multiple myeloma. Br. J. Haematol. 163, 478-486 (2013).

57. Kelly, K. R. et al. Indatuximab ravtansine (BT062) in combination with lowdose dexamethasone and lenalidomide or pomalidomide: clinical activity in patients with relapsed/refractory multiple myeloma. Blood 128, 4486-4486 (2016).

58. Touzeau, C., Moreau, P. \& Dumontet, C. Monoclonal antibody therapy in multiple myeloma. Leukemia 31, 1039 (2017)

59. Rosenblatt, J. \& Avigan, D. Targeting the PD-1/PD-L1 axis in multiple myeloma: a dream or a reality? Blood 129, 275-279 (2017).

60. Hallett, W. H., Jing, W., Drobyski, W. R. \& Johnson, B. D. Immunosuppressive effects of multiple myeloma are overcome by PD-L1 blockade. Biol. Blood Marrow Transplant. 17, 1133-1145 (2011).

61. Lesokhin, A. M. et al. Nivolumab in patients with relapsed or refractory hematologic malignancy: preliminary results of a phase lb study. J. Clin. Oncol. 34, 2698-2704 (2016).

62. Gorgun, $\mathrm{G}$. et al. Lenalidomide enhances immune checkpoint blockadeinduced immune response in multiple myeloma. Clin. Cancer Res. 21 4607-4618 (2015).

63. Mateos, M.-V. et al. Pembrolizumab in combination with lenalidomide and low-dose dexamethasone for relapsed/refractory multiple myeloma (RRMM): final efficacy and safety analysis. J. Clin. Oncol. 34, 8010-8010 (2016).

64. Badros, A. et al. Pembrolizumab, pomalidomide, and low-dose dexamethasone for relapsed/refractory multiple myeloma. Blood $\mathbf{1 3 0}$ 1189-1197 (2017).

65. Usmani, S. Z. et al. A phase 3 randomized study of pembrolizumab (pembro) plus lenalidomide (len) and low-dose dexamethasone (Rd) versus Rd for newly diagnosed and treatment-naive multiple myeloma (MM): KEYNOTE185. J. Clin. Oncol. 36, 8010-8010 (2018)

66. Ansell, S. et al. A phase 1 study of nivolumab in combination with ipilimumab for relapsed or refractory hematologic malignancies (CheckMate 039). Blood 128, 183-183 (2016).
67. Cho, H. J. et al. Atezolizumab in combination with daratumumab with or without lenalidomide or pomalidomide: a phase lb study in patients with multiple myeloma. Blood 132, 597-597 (2018).

68. Turtle, C. J., Hudecek, M., Jensen, M. C. \& Riddell, S. R. Engineered T cells for anti-cancer therapy. Curr. Opin. Immunol. 24, 633-639 (2012).

69. Lim, W. A. \& June, C. H. The principles of engineering immune cells to treat Cancer Cell 168, 724-740 (2017)

70. Till, B. G. et al. Adoptive immunotherapy for indolent non-Hodgkin lymphoma and mantle cell lymphoma using genetically modified autologous CD20-specific T cells. Blood 112, 2261-2271 (2008).

71. Mikkilineni, L. \& Kochenderfer, J. N. Chimeric antigen receptor T-cell therapies for multiple myeloma. Blood 130, 2594-2602 (2017).

72. Ali, S. A. et al. T cells expressing an anti-B-cell maturation antigen chimeric antigen receptor cause remissions of multiple myeloma. Blood $\mathbf{1 2 8}$ 1688-1700 (2016).

73. Obstfeld, A. E. et al. Cytokine release syndrome associated with chimericantigen receptor T-cell therapy: clinicopathological insights. Blood 130, 2569-2572 (2017)

74. Brudno, J. N. \& Kochenderfer, J. N. Toxicities of chimeric antigen receptor T cells: recognition and management. Blood 127, 3321-3330 (2016).

75. Raje, N. et al. Anti-BCMA CAR T-cell therapy bb2121 in relapsed or refractory multiple myeloma. N. Engl. J. Med. 380, 1726-1737 (2019).

76. Munshi, N. C. et al. Idecabtagene vicleucel (ide-cel; bb2121), a BCMA-targeted CAR T-cell therapy, in patients with relapsed and refractory multiple myeloma (RRMM): initial KarMMa results. J. Clin. Oncol. 38, 8503-8503 (2020).

77. Cohen, A. D. et al. B cell maturation antigen-specific CAR T cells are clinically active in multiple myeloma. J. Clin. Investig. 129, 2210-2221 (2019).

78. Berdeja, J. G. et al. Update of CARTITUDE-1: A phase Ib/II study of JNJ-4528, a B-cell maturation antigen (BCMA)-directed CAR-T-cell therapy, in relapsed/ refractory multiple myeloma. J. Clin. Oncol. 38, 8505-8505 (2020).

79. Mailankody, S. et al. Orvacabtagene autoleucel (orva-cel), a B-cell maturation antigen (BCMA)-directed CAR T cell therapy for patients (pts) with relapsed/ refractory multiple myeloma (RRMM): update of the phase 1/2 EVOLVE study (NCT03430011). J. Clin. Oncol. 38, 8504-8504 (2020).

80. Drent, E. et al. Feasibility of controlling CD38-CAR T cell activity with a Tet-on inducible CAR design. PLOS ONE 13, e0197349 (2018).

81. Sun, C. et al. Safety and efficacy of targeting CD138 with a chimeric antigen receptor for the treatment of multiple myeloma. Oncotarget 10, 2369-2383 (2019).

82. Maus, M. V. \& June, C. H. Making better chimeric antigen receptors for adoptive T-cell therapy. Clin. Cancer Res. 22, 1875-1884 (2016).

83. Paino, T. et al. Phenotypic identification of subclones in multiple myeloma with different chemoresistant, cytogenetic and clonogenic potential. Leukemia 29, 1186-1194 (2015).

84. Kadowaki, N. [Cancer therapy using bispecific antibodies]. [Rinsho ketsueki] Rinsho Ketsueki 59, 1942-1947 (2018).

85. Huehls, A. M., Coupet, T. A. \& Sentman, C. L. Bispecific T-cell engagers for cancer immunotherapy. Immunol. Cell Biol. 93, 290-296 (2015).

86. Shah, N., Chari, A., Scott, E., Mezzi, K. \& Usmani, S. Z. B-cell maturation antigen $(B C M A)$ in multiple myeloma: rationale for targeting and current therapeutic approaches. Leukemia 34, 985-1005 (2020).

87. Gramer, M. J. et al. Production of stable bispecific lgG1 by controlled Fab-arm exchange: scalability from bench to large-scale manufacturing by application of standard approaches. mAbs 5, 962-973 (2013).

88. Rader, C. DARTs take aim at BiTEs. Blood 117, 4403-4404 (2011)

89. Hipp, S. et al. A novel BCMAVCD3 bispecific T-cell engager for the treatment of multiple myeloma induces selective lysis in vitro and in vivo. Leukemia $\mathbf{3 1}$ 1743 (2016).

90. Topp, M. S. et al. Evaluation of AMG 420, an anti-BCMA bispecific T-cell engager (BiTE) immunotherapy, in $R / R$ multiple myeloma (MM) patients: Updated results of a first-in-human $(\mathrm{F} \mid \mathrm{H})$ phase I dose escalation study. J. Clin Oncol. 37, 8007-8007 (2019).

91. Costa, L. J. et al. First clinical study of the B-cell maturation antigen (BCMA) 2 +1 T cell engager (TCE) CC-93269 in patients (Pts) with relapsed/refractory multiple myeloma (RRMM): interim results of a phase 1 multicenter trial. Blood 134, 143-143 (2019).

92. Usmani, S. et al. Phase I study of teclistamab, a humanized B-cell maturation antigen (BCMA) x CD3 bispecific antibody, in relapsed/refractory multiple myeloma (R/R MM). J. Clin. Oncol. 38, 100-100 (2020). 
93. Cooper, D. et al. Safety and preliminary clinical activity of REGN5458, an antiBcma $\times$ anti-CD3 bispecific antibody, in patients with relapsed/refractory multiple myeloma. Blood 134, 3176-3176 (2019).

94. Catherine, P.-D. Tumour-associated antigens in multiple myeloma. Br. J. Haematol. 120, 3-9 (2003).

95. Espinoza-Delgado, I. Cancer vaccines. Oncologist 7(Suppl 3), 20-33 (2002).

96. Mayordomo, J. I. et al. Bone marrow-derived dendritic cells pulsed with synthetic tumour peptides elicit protective and therapeutic antitumour immunity. Nat. Med. 1, 1297-1302 (1995).

97. Rosenblatt, J. et al. Vaccination with dendritic cell/tumor fusion cells results in cellular and humoral antitumor immune responses in patients with multiple myeloma. Blood 117, 393-402 (2011).

98. Rosenblatt, J. et al. Vaccination with dendritic cell/tumor fusions following autologous stem cell transplant induces immunologic and clinical responses in multiple myeloma patients. Clin. Cancer Res. 19 3640-3648 (2013).

99. Rosenblatt, J. et al. PD-1 blockade by CT-011, anti PD-1 antibody, enhances ex-vivo $T$ cell responses to autologous dendritic/myeloma fusion vaccine. J. Immunother. 34, 409-418 (2011).

100. Rosenblatt, J. et al. Blockade of PD-1 in combination with dendritic cell/ myeloma fusion cell vaccination following autologous stem cell transplantation is well tolerated, induces anti-tumor immunity and may lead to eradication of measureable disease. Blood 126, 4218-4218 (2015).

101. Facon, T. et al. Phase 3 randomized study of daratumumab plus lenalidomide and dexamethasone (D-Rd) versus lenalidomide and dexamethasone (Rd) in patients with newly diagnosed multiple myeloma (NDMM) ineligible for transplant (MAIA). Blood 132, LBA-2-LBA-2 (2018).

102. Moreau, P. et al. Bortezomib, thalidomide, and dexamethasone with or without daratumumab before and after autologous stem-cell transplantation for newly diagnosed multiple myeloma (CASSIOPEIA): a randomised, openlabel, phase 3 study. Lancet 394, 29-38 (2019).

103. Voorhees, P. M., et al. Daratumumab, lenalidomide, bortezomib, \& dexamethasone for transplant-eligible newly diagnosed multiple myeloma: GRIFFIN. Blood. https://doi.org/10.1182/blood.2020005288 (2020).
104. Usmani, S. Z. et al. Carfilzomib, dexamethasone, and daratumumab versus carfilzomib and dexamethasone for the treatment of patients with relapsed or refractory multiple myeloma (RRMM): primary analysis results from the randomized, open-label, phase 3 study candor (NCT03158688). Blood 134 LBA-6-LBA-6 (2019).

105. Usmani, S. Z. et al. Clinical efficacy of daratumumab monotherapy in patients with heavily pretreated relapsed or refractory multiple myeloma. Blood $\mathbf{1 2 8}$ 37-44 (2016).

106. Attal, M. et al. Isatuximab plus pomalidomide and low-dose dexamethasone versus pomalidomide and low-dose dexamethasone in patients with relapsed and refractory multiple myeloma (ICARIA-MM): a randomised, multicentre, open-label, phase 3 study. Lancet $\mathbf{3 9 4}$ 2096-2107 (2019).

107. Moreau, P. et al. Isatuximab plus carfilzomib and dexamethasone vs carfilzomib and dexamethasone in relapsed/refractory multiple myeloma (Ikema): interim analysis of a phase 3, Randomized, Open-Label Study. EHA Library. Late Breaking Abstract LB2603 (2020).

108. Jakubowiak, A. et al. Randomized phase 2 study: elotuzumab plus bortezomib/dexamethasone vs bortezomib/dexamethasone for relapsed/refractory MM. Blood 127, 2833-2840 (2016).

109. Voorhees, P. M. et al. A phase 2 multicentre study of siltuximab, an antiinterleukin-6 monoclonal antibody, in patients with relapsed or refractory multiple myeloma. Br. J. Haematol. 161, 357-366 (2013).

110. Brudno, J. N. et al. T cells genetically modified to express an anti-B-cell maturation antigen chimeric antigen receptor cause remissions of poor-prognosis relapsed multiple myeloma. J. Clin. Oncol. 36 2267-2280 (2018)

111. Zhao, W.-H. et al. A phase 1, open-label study of LCAR-B38M, a chimeric antigen receptor $T$ cell therapy directed against B cell maturation antigen, in patients with relapsed or refractory multiple myeloma. J. Hematol. Oncol. 11 141-141 (2018).

112. Xu, J. et al. Exploratory trial of a biepitopic CAR T-targeting B cell maturation antigen in relapsed/refractory multiple myeloma. Proc. Natl Acad. Sci. USA. 116, 9543-9551 (2019) 